\title{
The potential added value of FDG PET/CT for COVID-19 pneumonia
}

\author{
Yan Deng ${ }^{1} \cdot$ Lei Lei $^{2} \cdot$ Yue Chen ${ }^{2} \cdot$ Wei Zhang $^{2}$
}

Received: 2 March 2020 / Accepted: 10 March 2020 / Published online: 21 March 2020

(C) Springer-Verlag GmbH Germany, part of Springer Nature 2020

Dear Sir,

By February 27, 2020, COVID-19 had infected about 82,163 people and killed 2800 in about 37 countries and regions [1]. The coronavirus, like SARS-CoV-2 and MERS$\mathrm{CoV}$, belongs to the coronavirus family [2]. Most infections are followed by fever, cough, and other non-specific symptoms. Mild symptoms were similar to the common cold, mortality was lower in relatively healthy and younger patients, and most patients without complications survived the infection and fully recovered. Due to the progression of pneumonia and a pathologic disease characterized by diffuse alveolar damage, severe cases are most likely leading to adult respiratory distress syndrome (ARDS) [3].

Nucleic acid testing (RT-PCR) is the gold standard for the diagnosis of COVID-19 infection, but with a high false negative rate, which is easy to miss the diagnosis and cause the spread of the epidemic [4]. Chest high-resolution CT is the routine-preferred method for screening, diagnosis, course severity assessment, and efficacy monitoring of COVID-19 pneumonia. CT findings of COVID-19 pneumonia have been widely reported [5-7]. In the early stage, single or multiple plaques, masses, nodules, or segmental ground glass density lesions were found in the lung. As the disease progressed, the lesions increased and spread to the center. The inflammatory exudation, consolidation, and density increased, accompanied by pulmonary vascular shadow thickening, air bronchi sign, paving stone sign, interlobular septal thickening, pleural

This article is part of the Topical Collection on Infection and inflammation

Wei Zhang

zhangwscd@163.com

1 Department of Pediatrics, Affiliated Hospital of Southwest Medical University, Luzhou, Sichuan, People's Republic of China

2 Department of Nuclear Medicine, Affiliated Hospital of Southwest Medical University/Nuclear Medicine and Molecular Imaging Key Laboratory of Sichuan Province, No 25 TaiPing St, Jiangyang District, Luzhou 646000, Sichuan, People's Republic of China effusion, and other signs. The images are similar to those seen in viral pneumonia such as MERS-CoV and SARS-CoV-2 [8].

As for the FDG PET/CT imaging results after COVID-19 infection, there are only four patients with highly suspected COVID-19 infection having been reported so far by Qin et al. [9]. They found lung lesions characterized by increased FDG uptake and evidence of lymph node involvement. In fact, as a non-invasive imaging method, FDG PET/CT plays an important role in evaluating inflammatory and infectious pulmonary diseases, monitoring disease progression and treatment effect, and improving patient management [10]. When the virus infects the body, the cascade of reactions activates inflammatory cells such as neutrophils, monocytes, and effector T cells by releasing local chemokines. In acute inflammation or chest infection, activated neutrophils are heavily dependent on anaerobic glycolysis, requiring increased glucose and resulting in high FDG uptake [11]. Granulocytes and macrophages also facilitate glucose transport under chronic conditions. Before FDG uptake was observed in patients with COVID-19 infection by Qin et al. [9], Das et al. also observed significant FDG uptake in patients with MERS-CoV infection which progressed to pneumonia [12]. Chefer et al. used FDG PET/ $\mathrm{CT}$ to observe the rhesus macaques model and to simulate the host response after MERS-CoV infection [13]. In their model, increased FDG uptake was observed in mediastinal and axillary lymph nodes, and the uptake variation constant was associated with increased monocytes percentage. It is not hard to see the reason. Increased monocytes in lymphoid tissue used the immune system to fight off viral infections, and monocytes contributed to increase uptake of FDG. As for lymph node enlargement, it has been manifested in pneumonia caused by parainfluenza virus and adenovirus. Although lymph node enlargement on $\mathrm{CT}$ is rare, lymphadenopathy is present in more than $1 \%$ of patients [14]. Considering that $\mathrm{CT}$ is less sensitive to host reactions than FDG PET/CT, the actual percentage of lymph node involvement may be higher.

FDG uptake is often thought of as non-specific inflammatory or immune activation that fails to reflect the host response produced by specific cells. Depending on the stages of the 
virus and diseases, specific immune cell populations are involved in the response. The role of FDG PET/CT should be reflected in changes in uptake patterns and locations during virus exposure, which may be useful for monitoring the effects of treatment. From Qin et al.'s paper [9], we can roughly see that patients with higher FDG uptake in lung lesions take longer to heal and are positively correlated with the value of the erythrocyte sedimentation rate. Another advantage of FDG PET/CT is that it may be more appropriate when viral infections are associated with other factors. As the disease progresses, it may cause damage to the gastrointestinal tract, kidneys, heart, bone marrow, and other organs [15]. In fact, in Chefer's MERS-CoV animal model, high bone marrow uptake over a long period of time has been observed [13]. FDG $\mathrm{PET} / \mathrm{CT}$ has the potential to add value to the challenge of diagnosing complications caused by viruses such as COVID-19.

As mentioned above, FDG PET/CT is a sensitive method to detect and monitor inflammatory diseases, such as viral pneumonia, monitor disease progression and treatment outcomes.

Funding information This study was funded by the National Natural Science Foundation of China (grant number 81701739).

\section{Compliance with ethical standards}

Conflict of interest The authors declare that they have no conflict of interest.

Ethical approval This article does not contain any studies with human participants or animals performed by any of the authors.

\section{References}

1. Coronavirus COVID-19 Global Cases by Johns Hopkins CSSE. https://gisanddata.maps.arcgis.com/apps/opsdashboard/index. html\#/bda7594740fd40299423467b48e9ecf6. Accessed 27 Feb 2020.

2. Park M, Thwaites RS, Openshaw PJM. COVID-19: lessons from SARS and MERS. Eur J Immunol. 2020;50(3):308-311.
3. Huang C, Wang Y, Li X, Ren L, Zhao J, Hu Y, et al. Clinical features of patients infected with 2019 novel coronavirus in Wuhan, China. Lancet. 2020;395:497-506.

4. Lan L, Xu D, Ye G, Xia C, Wang S, Li Y, et al. Positive RT-PCR test results in patients recovered from COVID-19. JAMA. 2020. https:// doi.org/10.1001/jama.2020.2783.

5. Kanne JP, Little BP, Chung JH, Elicker BM, Ketai LH. Essentials for radiologists on COVID-19: an update radiology scientific expert panel. Radiology. 2020295(1);16-17.

6. Shi H, Han X, Jiang N, Cao Y, Alwalid O, Gu J, et al. Radiological findings from 81 patients with COVID-19 pneumonia in Wuhan, China: a descriptive study. Lancet Infect Dis. 2020. https://doi.org/ 10.1016/S1473-3099(20)30086-4

7. Lee EYP, Ng MY, Khong PL. COVID-19 pneumonia: what has CT taught us. Lancet Infect Dis. 2020. https://doi.org/10.1016/S14733099(20)30134-1

8. Koo HJ, Lim S, Choe J, Choi SH, Sung H, Do KH. Radiographic and CT features of viral pneumonia. Radiographics. 2018;38:719 39.

9. Qin C, Liu F, Yen TC, Lan X. ${ }^{18}$ F-FDG PET/CT findings of COVID-19: a series of four highly suspected cases. Eur J Nucl Med Mol Imaging. 2020. https://doi.org/10.1007/s00259-02004734-w .

10. Capitanio S, Nordin AJ, Noraini AR, Rossetti C. PET/CT in nononcological lung diseases: current applications and future perspectives. Eur Respir Rev. 2016;25:247-58.

11. Jones HA, Marino PS, Shakur BH, Morrell NW. In vivo assessment of lung inflammatory cell activity in patients with COPD and asthma. Eur Respir J. 2003;21:567-73.

12. Das KM, Lee EY, Langer RD, Larsson SG. Middle East respiratory syndrome coronavirus: what does a radiologist need to know. AJR Am J Roentgenol. 2016;206(6):1193-201.

13. Chefer S, Thomasson D, Seidel J, Reba RC, Bohannon JK, Lackemeyer MG, et al. Modeling [(18)F]-FDG lymphoid tissue kinetics to characterize nonhuman primate immune response to Middle East respiratory syndrome-coronavirus aerosol challenge. EJNMMI Res. 2015;5:65.

14. Xu X, Yu C, Qu J, Zhang L, Jiang S, Huang D, et al. Imaging and clinical features of patients with 2019 novel coronavirus SARSCoV-2. Eur J Nucl Med Mol Imaging. 2020. https://doi.org/10. 1007/s00259-020-04735-9.

15. Gao QY, Chen YX, Fang JY. 2019 novel coronavirus infection and gastrointestinal tract. J Dig Dis. 2020. https://doi.org/10.1111/17512980.12851 .

Publisher's note Springer Nature remains neutral with regard to jurisdictional claims in published maps and institutional affiliations. 